\title{
Novel de novo heterozygous mutation on SYNGAP1 from the Indian population
}

\section{CURRENT STATUS: POSTED}

Research Square

\section{Vijaya Verma}

Jawaharlal Nehru Centre for Advanced Scientific Research CSIR Centre of Excellence in Chemistry

Amit Mandora

syngap1 research foundation

Abhijeet Botre

KEM hospital

James Premdoss Clement

Jawaharlal Nehru Centre for Advanced Scientific Research

ฯ clement@jncasr.ac.inCorresponding Author

ORCiD: https://orcid.org/0000-0001-7625-6430

\section{DOI:}

10.21203/rs.2.22020/v1

\section{SUBJECT AREAS}

Internal Medicine

\section{KEYWORDS}

SYNGAP1, autism spectrum disorder, intellectual disability, epilepsy 
Abstract

Background : Exome sequencing is a prominent tool to identify novel and deleterious mutations which could be nonsense, frameshift, and canonical splice-site mutations in a specific gene. De novo mutations in SYNGAP1, which codes for synaptic RAS-GTPase activating the protein, causes Intellectual disability (ID) and Autism Spectrum Disorder (ASD). SYNGAP1 related ASD/ID is one of the rare diseases that is detrimental to the normal neuronal developmental and disrupts the global development of a child.

Results: We report a case of a child of 2-year old with global developmental delay, microcephaly subtle dysmorphism, absence seizures, disrupted sleep, delay in learning a language, and eating problems. Upon further validation, the child has a few traits of ASD. Here, based on focused exome sequencing, we report a de novo heterozygous mutation in SYNGAP1 exon 11 with c. 1861 C>T (p.arg612ter). Currently, the child is on atorvastatin and has shown considerable improvement in global behaviour and cognitive development. The long-term follow up of the child's development would contribute to the already existing knowledge of the developmental trajectory in individuals with SYNGAP1 heterozygous mutation.

Conclusion: In this report, we discuss the finding of a novel mutation in one of the genes, SYNGAP1 , implicated in ASD/ID. In addition, we discuss the current treatment prescribed to the patient and the progress of global developmental of the child.

\section{Full-text}

Due to technical limitations, full-text HTML conversion of this manuscript could not be completed. However, the manuscript can be downloaded and accessed as a PDF. 